\title{
Geriatric pain management, pharmacological and nonpharmacological considerations
}

\author{
Alan David Kaye ${ }^{1}$, Amir R. Baluch², Rachel J. Kaye ${ }^{1}$, Rashid S. Niaz ${ }^{1}$, Aaron J. Kaye ${ }^{3}$, Henry \\ $\mathrm{Liu}^{4}$, and Charles J. Fox ${ }^{5}$ \\ 1. Louisiana State University School of Medicine, New Orleans, LA, USA \\ 2. Metropolitan Anesthesia Consultants, Dallas, TX, USA \\ 3. Stanford University, Palo Alto, CA, USA \\ 4. Tulane School of Medicine, New Orleans, LA, USA \\ 5. Louisiana State University School of Medicine, Shreveport, LA, USA
}

\begin{abstract}
Persistent pain is prevalent in the elderly population, although it is not an inevitable part of aging. It is important to understand how to manage pain effectively in old age, particularly because an increasing number of individuals are becoming older, or living longer. Several problems, less common in younger adults, may complicate the treatment of pain. An accurate pain assessment is required for the most efficient strategy of pain treatment. Challenges to an effective pain assessment include: pain underreporting by patients, atypical manifestations of pain in elderly, age-associated pharmacodynamic and pharmacokinetic changes to specific drugs, other general age-related changes, and misconceptions about tolerance or addiction to opioids. However, physicians are able to provide geriatric patients with appropriate analgesia by using comprehensive assessment involving a multidisciplinary approach, and the appropriate use of various treatment modalities.Keywords: geriatric pain management, pain assessment, pain threshold, geriatric pharmacokinetics
\end{abstract}

Received 11 August 2011; received in revised form 26 June 2013; accepted 20 August 2013. Available online 07 March 2014.

\section{Introduction}

Elderly men and women represent the fastest growing demographic in the world's population. By 2040 the number of people aged 65 and older worldwide will increase to 1.3 billion from the current 506 million. In the United States alone, the elderly population will increase from 40 million to 80 million by 2040 . Although pain is not part of normal aging, a significant proportion of the elderly experience physical discomfort. Studies

Alan David Kaye, MD, PhD, Professor and Chairman, Department of Anesthesiology, Louisiana State University School of Medicine, New Orleans, Louisiana. Amir R. Baluch, MD, Attending Anesthesiologist, Metropolitan Anesthesia Consultants, Dallas, Texas. Rachel J. Kaye, and Rashid S. Niaz, MD, Research Associate, Department of Anesthesiology, Louisiana State University School of Medicine, New Orleans, Louisiana. Aaron J. Kaye, undergraduate, Stanford University, Palo Alto, California. Henry Liu, MD, Associate Professor, Department of Anesthesiology, Tulane Medical Center, New Orleans, Louisiana. Charles J. Fox, MD, Professor and Chairman, Department of Anesthesiology, Louisiana State University School of Medicine, Shreveport, Louisiana. Correspondence regarding this article should be directed to: Dr. Alan David Kaye, MD, PhD, Professor and Chair, Department of Anesthesiology, LSU School of Medicine, 1542 Tulane Ave, Room 656, New Orleans, Louisiana 70112. Phone: 504-568-2319. E-mail: akaye@1suhsc.edu suggest $25 \%$ to $50 \%$ of community-dwelling elders report pain, and that for elderly residents in nursing homes the prevalence of pain is as high as $80 \%$ (Kaye, Baluch, \& Scott, 2010; Charette \& Ferrell, 2007; Berdine \& Mihalyo, 2004).

Chronic geriatric pain is defined as "an unpleasant sensory and emotional experience associated with actual or potential tissue damage or described in terms of such damage for persons who are either aged (65 to 79 years old) or aged (80 and over) and who have had pain for greater than 3 months." (Kaye et al., 2010) With inadequate treatment, this pain produces functional impairment and decreased quality of life for elderly patients. Other consequences may include sleep disturbances, depression, anxiety, decreased socialization, cognitive dysfunction, polypharmacy, malnutrition, and increased costs associated with health care (Kaye et al., 2010; Manchikanti, Singh, Datta, Cohen, \& Hirsch, 2009; Bruckenthal, 2008). Pain-related lessened activity may also lead to gait impairments, which may result in injuries from falls and accidents.

The prevalence of chronic pain increases with age (Crook, Rideout, \& Browne, 1984) along with neuralgias and joint pain (Badley \& Tennant, 1992). A significant proportion of elderly individuals experience daily pain but fail to receive adequate treatment. In one study of 
elderly cancer patients with pain, 21\% between 65 and 74 years of age received no pain medication; in those 75 to 84 years old, $26 \%$ received no pain medication, and for those above the age of $84,30 \%$ received no pain medication (Kaye et al., 2010; Cleeland, 1998). Undertreatment is exacerbated further by a lack of efficient methods for detection and management of geriatric pain (Woo, Ho, Lau, \& Leung, 1994). A study of elderly nursing home residents found $66 \%$ with pain; however, physician assessment detected only $34 \%$ (Kaye et al., 2010; Sengstaken \& King, 1993; Ferrell, 1991).

\section{Barriers to effective pain assessment in elderly patients}

Geriatric pain assessment is a complex clinical process influenced by several factors. Complicating factors include cognitive or language impairments that diminish communicative abilities, coexisting illnesses, concomitant medications, and the cost of drugs. Assessment can be impacted specifically by negative mood states such as depression, heightened anxiety, psychosocial concerns, poor memory, and failing health. Elderly patients may underreport discomfort, thinking that pain is an inevitable part of aging or because they believe their physician is too busy to hear such concerns. Additionally, the elderly may fear the meaning of the pain and worry about the possible side effects of medications. Patients and healthcare providers alike may also avoid the use of opioids because of misconceptions about addiction and tolerance.

There are needs for more reliable and valid methods of identifying, diagnosing, and understanding pain in older individuals. These problems have been the focus of an increasing number of papers related to pain measurement in patients with dementia, in addition to pain assessment research more generally (Kaye et al., 2010; Ferrell, Ferrell, \& Rivera, 1995; Kovach, Weissman, Griffie, Matson, \& Muchka, 1999; Warden, Hurley, \& Volicer, 2003).

\section{Pain assessment and the physical examination}

Geriatric pain assessment requires a comprehensive and multidisciplinary approach to the description, diagnosis, and management of chronic pain. Physicians, psychologists, psychiatrists, and physical therapists work together to develop pain management strategies based on their individual evaluations of the patients. Meetings with patient caretakers, as well as laboratory or imaging studies, may be necessary if the initial workup does not provide sufficient information for a diagnosis.

Evaluations of patient functional abilities and performances are included in the assessment. Impairments in functional status may impact degree of independence, quality of life, and need for caretakers. Assessment of patient daily activities and habits such as using Activities of Daily Living (ADL) and
Instrumental Activities of Daily Living (IADL) ferrets out possible functional limitations. This information can then be incorporated into treatment plans toward the goals of maximizing patient function and decreasing pain perception (Kaye et al., 2010).

\section{Pain assessment scales}

Verbal Descriptor Scales (VDS), Numerical Rating Scales (NRS), and Visual Analogue Scales (VAS) are commonly used to assess pain intensity. Current data support the validity of all three methods. The VDS has been recommended specifically for use with elderly patients, and patients often prefer it to the other measures because it is the easiest to complete (Herr, Spratt, Mobily, \& Richardson, 2004). Because studies have shown an association between the VAS and unscorable or incorrect responses in the elderly, the measure is used best with caution (Gagliese, 2002; Gagliese, \& Melzack, 1997). However, in clinical and research settings the VAS has proven reliable and provides the advantages of simplicity, easy administration, and minimal obtrusiveness (Kaye et al., 2010). Difficulty has been reported in elderly patients with answering the NRS, particularly when administered verbally (Kaye et al., 2010; Herr \& Mobily, 1993; Benesh, Szigeti, Ferraro, Gullicks, 1997; Gagliese \& Melzack, 1997).

The McGill Pain Questionnaire (MPQ) assesses the sensory, affective, evaluative, and miscellaneous components of pain (Melzack, 1975). The validity, reliability, and discriminative capabilities of the scale are supported and are not age-related. Pain may be described in terms of intensity, affect, location, or several other domains (Ferrell, 2000). However, the MPQ tends to be time-consuming and difficult to use in clinical settings.

\section{Pain history and examination}

In addition to the evaluation of pain intensity and its characteristics, a thorough examination is needed. A detailed history is taken, and a physical examination is performed including a review of systems and relevant laboratory data, imaging studies, and diagnostic tests. An in-depth review of the location, intensity, and other factors associated with reported pain is conducted. Baseline functionality is determined by evaluation of ADLs, balance, and gait. Additionally, evaluation of patient psychological functioning and present social and living situations is conducted. The temporal relationship among significant life events, medical interventions, and various pain and emotional complaints are noted.

Any self-reported pain is thoroughly explored. Pain that is persistent, recurrent, and impacts patient quality of life or functional capacity is considered significant. Many elderly do not complain specifically of "pain", but instead they use a variety of terms such as "hurting", "burning", or "aching" to refer to their problems. As a result, terms other than "pain" may be targeted to screen 
for symptoms. Behavioral cues such as vocalizations and changes in behavioral or physical functioning can indicate the presence and severity of pain. These are primarily important in the assessment of individuals with cognitive impairments. Reports from caregivers and close relatives are essential, especially in patients with cognitive or language impairment.

\section{History}

If pain symptoms have increased from the previous visit or treatment session, the physician should increase the frequency of assessment using clinical judgment. Since the older population commonly has multiple medical problems, it is not uncommon to find multiple sources of pain. The intensity, character, frequency, location, and duration of pain are included in the history. The physician should determine whether symptoms are due to one underlying pathology or multiple overlapping pathologies. Factors that exacerbate or ameliorate pain can help to elucidate the diagnosis. Therefore, questions related to effects of past treatments as well as a thorough analgesic history should be asked. After determining these factors, inquiry should be made concerning the patient's response to current treatments and subsequent effects on the patient's physical, mental, social, and functional status. In addition, a quantitative assessment of patient pain is obtained with the most appropriate measurement scale. To the extent possible, physicians review the previous medical, surgical, and psychiatric condition daily, as well as records of injuries and accidents. Coexisting diseases along with current and previous treatments can impact treatment decisions.

It is not uncommon for an older patient with pain to have anxiety or depression or both, and oftentimes it is unclear if chronic pain is causing depression or anxiety or if depression and anxiety are causing chronic pain. A depression assessment using the Geriatric Depression Scale (GDS) may be used in these situations to help determine which occurred first. The medication history consists of listing all current and past medications, dosages, and adverse effects, and prescribed, over-thecounter, and herbal supplements are included.

\section{Physical examination}

The physical exam assesses the neuromuscular systems with attention to impairments, weakness, numbness, tingling, hyper/hypoalgesia, hyperpathia, or allodynia. Certain pathologies may be suggested by the presence of local inflammation, bony deformities, or trigger points at specific sites. Gait and balance are also assessed to determine functional mobility.

\section{Functional assessment}

Physical function is determined by assessing patient ability to perform ADLs. Mobility impairments may contribute to deficits in physical functioning and are evaluated by gait, balance, and range of motion testing.

\section{Psychosocial assessment}

Patient psychosocial functioning can be determined by exploring factors including mood, social support systems, family or caregiver relationships, and financial resources. Heightened anxieties and mild to severe depression symptoms are common in individuals with chronic pain and behaviors associated with anxiety or depressive disorders are noted. The availability and type of relationship in social support systems are explored. Patients with greater interpersonal involvement and more supportive social contacts spend less time focusing on their painful condition and consequently can exhibit greater reductions in pain. Conversely, interpersonal isolation and negative social relationships may impede treatment outcome.

\section{Follow-up interval}

Once the initial assessment has been completed, a follow-up interval is determined in relation to the severity of the pain and dysfunction. Follow-up visits aid in reassessing improvement or worsening of the condition, patient compliance, and complications with medications. Evaluators observe and record the positive and negative effects of the therapeutic modalities and analgesics and modify the treatment plan accordingly (American Geriatrics Panel on chronic pain in older persons, 1998).

\section{Pain assessment in the cognitively impaired}

Pain assessment is more challenging in individuals with cognitive impairments. These patients may lack the capacity to report pain accurately and reliably, although cognitively impaired individuals who have retained adequate communication skills are more likely to provide physicians with a valid pain report. Research suggests that self-reports from patients characterized by mild to moderate cognitive impairments are as valid and reliable as reports from those with normal cognitive functions (Parmelee, Smith, \& Katz, 1993; Weiner, Peterson, Logue, \& Keefe, 1998). As a result, these mildly to moderately impaired individuals can be assessed similarly to patients with normal cognitive function, beginning with a self-report and thorough examination. The VDS is generally recommended for measuring pain intensity in patients with mild to moderate cognitive impairment (Herr \& Mobily, 1993; Herr \& Garand, 2001).

The assessment of pain in individuals who exhibit cognitive impairments and/or frank deficits in receptive and expressive language poses major challenges. There is currently no clearly best method or guidelines for evaluating pain in these patients. Behavioral cues may be used to evaluate pain in non-communicative patients, including observing vocalizations, facial expressions, withdrawn/agitated behavior, and changes in gait or posture. Nevertheless, studies show many of these assessments underrate pain intensity (Pautex et al., 2005; Pautex et al., 2006). It may be helpful for patient caregivers or close relative to provide reports containing information regarding patient pain and observed changes 
in behavior and emotional responsiveness, although there are limits to these assessments (Ferrell, 1991; Lin, Lum, Mehr, \& Kane, 2006).

\section{Pathophysiological changes in the elderly}

During normal aging there is a steady reduction of organ system function and homeostatic functions. The central nervous system, hepatic, renal, and respiratory systems are the most important organ systems affected with aging.

\section{Central Nervous System}

Elderly patients present a higher prevalence of neurological diseases and dysfunctions resulting from or associated with age-induced changes in the central nervous system (CNS). Mental processing deficiencies are present in $15 \%$ of the total geriatric population, whereas $50 \%$ of elderly subjects who live in nursing homes have either a psychological illness or dementia (Ferrell, 1996). Other conditions include strokes, transient ischemic attacks, and movement disorders, which may affect pain assessment and treatment efficacy (Kaye et al., 2010; Broe, 1989).

In many patients seeking pain relief, the number of neurotransmitter cell receptors decreases with ageassociated cortical and subcortical atrophy of brain tissue. Synaptic efficiency decreases as neurons fail to regenerate and are replaced by proliferating glial cells (Long, 1985). The greatest loss of neurons is observed in the locus coeruleus and substantia nigra and has an effect on pain transmission (Ghafoor, 2003). Reduction in dendritic synapses and intracellular enzymes additionally contribute to the decreased synaptic ability (Kaye et al., 2010; Coleman \& Flood, 1987).

Age-induced changes in the CNS are responsible for deficits in cognitive and motor functions and may occur as early as 50 years of age. The development and onset of CNS conditions are influenced by important factors such as heredity and genetics, concomitant disease, level of intellectual complexity or ability, and stresses from daily activities or trauma (Samorajski, 1976).

Neuroendocrine function decreases with age and contributes to common neurological diseases present in elderly adults. Parkinson's disease and Alzheimer's disease show reduced dopaminergic and cholinergic/ hypothalamic/noradrenergic function, respectively (Rehman \& Masson, 2001).

Minor alterations in memory resulting in neurological changes are thought to be a normal feature of aging. However, dementia is present in $5 \%$ to $20 \%$ of adults age 65 and older. Alzheimer's disease is the most common cause of dementia, constituting $60 \%$ of all cases. There are other possible causes or corollaries of dementia that require exploration including Parkinson's disease, vascular disorders, normalpressure hydrocephalus, neoplastic diseases, alcoholism and other long-term additions, genetically transmitted neurodegenerative diseases, CNS infections, metabolic disorders, and pseudodementias (Kaye et al., 2010; Simon, Aminoff, \& Greenberg, 1989).

\section{Hepatic}

An elderly liver may prolong the clearance of drugs from the body secondary to prehepatic, intrahepatic, or posthepatic causes. Prehepatic dysfunction includes decreased first-pass metabolism and blood extraction, which may be secondary to reduced arterial and portal blood flow and lower gastrointestinal absorption. Intrahepatic dysfunction may be caused due to hepatocellular pathology, for example, cirrhosis. Additionally, posthepatic dysfunction is caused by either biliary tree or enterohepatic circulation pathology (Kaye et al., 2010).

\section{Renal system}

Starting at the age of 40 , renal function declines $\sim 1 \%$ per year or a $1 \mathrm{~mL} / \mathrm{min} / \mathrm{yr}$ decline in creatinine clearance (Kaye et al., 2010; Stiff, 1993). In healthy elderly individuals, however, the overall function of the kidneys appears to be maintained (Kohli et al., 2006). In a recent report, more than $5 \%$ of elderly patients were determined to exhibit renal failure, primarily as a result of diabetic nephropathy.

\section{Pain threshold}

Several studies have focused on the relationship between aging and pain threshold. Gibson (2003) conducted a meta-analysis of 50 previous studies that examined age differences in sensitivity to induced pain. Of these 50 studies, 21 reported data that supported an increase in pain threshold with increasing age, three reported a decrease, and 17 reported no difference. The effect size was $0.074(p<0.0005)$, indicating evidence of an increase in pain threshold with advancing age (Kaye et al., 2010; Gibson, 2003).

Further studies have shown that the effect of age on pain threshold depends on the method of pain induction. Lautenbacher, Kunz, Strate, Nielsen, and ArendtNielsen (2005) compared pain perception from different stimuli in two groups. Each consisted of 20 men classified according to age, with the mean age of the older group 71.6 and the younger at 27.1. Results revealed that for older individuals somatosensory thresholds for non-noxious stimuli were increased, whereas pressure thresholds decreased. No age-associated changes were observed for heat pain threshold (Lautenbacher et al. 2005). These results confirm previous studies (Kaye et al., 2010; Gibson, 2003; Lin, Hsieh, Chao, Chang, \& Hsieh, 2005; Verrillo, Bolanowski, \& Gescheider, 2002; Woodrow, Friedman, Siegelaub, Collen, 1972; Pickering, Jourdan, Eschalier, \& Dubray, 2002).

The difference in pain perception may be related to age-associated changes in CNS. Research has focused primarily on the differential changes in $\mathrm{A} \delta$ - and $\mathrm{C}$-fibers that transmit epicritic/phasic pain and protophasic/tonic pain, respectively. Results from these studies support the presence of a deficit in the ability of older people to 
perceive $\mathrm{A} \delta$-mediated pain, but there was no evidence of age-related differences in the perception of C-fiber mediated pain (Chakour, Gibson, Bradbeer, \& Helme, 1996).

\section{Common comorbidities in the elderly}

The prevalence of certain pain-associated conditions increases with age. Comorbidity represents an important consideration in the assessment and treatment of pain. The most effective treatments are based on a solid base of knowledge concerning common geriatric pain conditions, thus allowing delivery of the most efficient treatments. A list of common geriatric pain syndromes is shown in Box 1.

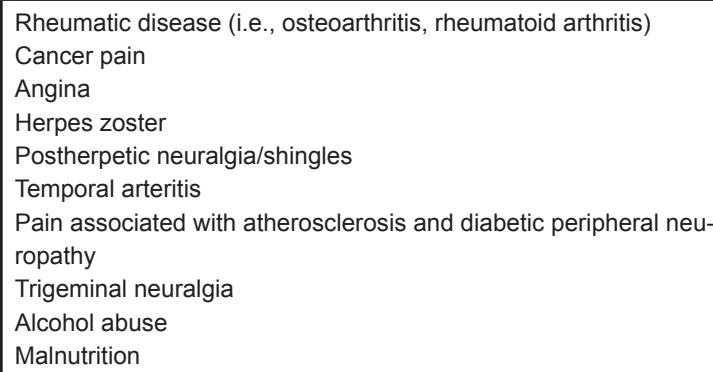

Box 1. Description of chronic geriatric pain syndromes

Modified from Kaye A. D., Baluch A., \& Scott J. T. (2010). Pain management in the elderly population: A Review. The Ochsner Journal, 10, 183.

\section{Pharmacokinetic changes}

Increased body fat, decreased total body water, and decreased muscle mass are age-related changes in body composition that effect drug distribution (Kaye et al., 2010; Turnheim, 2003; Vuyk, 2003).

Diuretics may further decrease body water, and these drugs are commonly in the elderly. Hydrophilic drugs such as ethanol, lithium, and cimetidine are less used, distributed effectively, and result in more rapid onset of action and increased peak concentrations. Consequently, there is a greater risk of side effects for hydrophilic medications in the elderly. Lipophilic drugs such as diazepam and lidocaine have an increased distribution and slow onset of action. With repeated dosing, accumulation of lipophilic drugs will occur. Frailty and malnourishment are common in elderly patients and additionally influence drug distribution (Kaye et al., 2010; ElDesoky, 2007).

Decreases in serum albumin concentrations increase the proportion of free drug in the plasma. Therefore, highly protein bound drugs such as NSAIDs, benzodiazepines, and anti-epileptics will cause higher levels of adverse effects in elderly patients. Poor nutritional status may also affect free drug concentrations (Kaye et al., 2010).

Age-associated decreases in liver size and hepatic blood flow reduce the clearance of drugs that undergo first-pass metabolism in the liver such as lidocaine and narcotics (Bresler \& Bahl, 2003). The amount of free drug in the plasma is increased and, as a consequence, elderly patients are at risk for a greater chance of side effects and adverse reactions. The presence of frailty, comorbidities, or polypharmacy can additionally increase these chances (Kitani, 1986; Kinirons \& Crome, 1997). To avoid complications in the elderly, drugs that undergo significant first-pass metabolism should be initiated slowly at low doses (Crooks \& Stevenson, 1981). The renal elimination of drugs and their metabolites is reduced with age as a result of decreased glomerular filtration and tubular reabsorption and decline in renal mass and blood flow (Shi, Mörike, \& Klokz, 2008). Drugs that are eliminated primarily renal, including several antibiotics and lithium, will have increased drug levels for a prolonged duration (Wright \& Warpula, 2004). Patients experiencing ageassociated renal insufficiency and dehydration should be cautiously administered renally cleared drugs, and the dosage should be decreased while the frequency of administration increased. Overall reduction in kidney and liver function increases the half-life of many benzodiazepines and tricyclic antidepressants.

Hepatic phase I oxidation metabolism including oxidation, reduction, and hydrolysis is more susceptible to age than hepatic phase II conjugation reactions such as acetylation, glucuronidation, sulfation, and glycine conjugation (Wright \& Warpula, 2004). Drugs that undergo phase I metabolism have reduced elimination in the elderly. Acetaminophen and diazepam are metabolized at the same rate for all ages. However, drugs metabolized by the same enzyme system including carbamezapine, lidocaine, and fentanyl undergo reduced metabolism in the elderly. Glucuronidation of morphine and glutathione conjugation of acetaminophen are phase II reduction reactions and are not affected by age. To decrease side effects that are a result of reduced metabolism and elimination, physicians should be cautious when administering drugs that undergo hepatic phase I metabolism (Kaye et al., 2010).

\section{Pharmacodynamic changes}

In the elderly, the decline in drug sensitivity is associated strongly with age-related decline in CNS function (Kaye et al., 2010). Geriatric patients exhibit increased sensitivity to drugs that act centrally or have centrally mediated effects including benzodiazepines, opioids, and psychotropic drugs. As a result, these drugs produce exaggerated effects in older individuals. In contrast, the adrenergic and cholinergic autonomic nervous system has reduced sensitivity to receptorspecific drugs with age. Reduced response to $\beta-$ adrenergic stimulation results the decreased effect of $\beta$-blockers (Muravchick, 1998).

\section{Observance to physician in the elderly population}

Poor medical compliance prevents physicians from providing effective pain management and can be attributed to multiple factors. Factors include poor physician-patient communication, cost of care, ethnic 
and cultural beliefs, drug and dosage form, and insurance coverage (Kaye et al., 2010; Steinman \& Hanlon, 2010). Approaches to involved efforts to increase compliance include communicating the purpose and side effect profile of medications, prescribing simple dosing regimens, decreasing the total number of drugs needed, scheduling regular follow-ups, preparing individualized medication cards, establishing medication review by pharmacists, and using of multi-compartment dose administration aids (George, Elliott, \& Steward, 2008). Many studies have been conducted to determine the best way to address non-compliance; however, inconsistent study methodologies and findings have prevented the drawing of firm conclusions in favor of any specific method over another.

\section{Pain management options in the elderly}

A multidisciplinary approach to pain management is recommended to provide the most effective and comprehensive addressing of all the components of pain. Pain treatment modalities for the elderly may be classified into the following four groups (Kaye et al., 2010):

1. Pharmacotherapy

2. Physical rehabilitation

3. Psychological support

4. Interventional procedures

\section{Pharmacotherapy}

Pharmacotherapy is the most common method used to control geriatric pain and, in addition, is relatively easy to implement. Drug treatment consists of NSAIDs, muscle relaxants, opioids, and adjuvant therapy. Analgesic drugs are effective and safe when properly used; however, the elderly have an increased risk of adverse reactions. The cognitive, functional, and physiological status of the patient may be affected. The American Geriatric Society (AGS) and the World Health Organization (WHO) have formed counsels to conceptualize and develop better approaches to managing geriatric pain (Kaye et al., 2010; American Geriatrics Society Panel on Pharmacological Management of Persistent Pain in Older Persons, 2009).

\section{A. Nonopioids}

I. Acetaminophen is considered as initial and ongoing pharmacotherapy in the treatment of persistent pain, particularly musculoskeletal pain, owing to its demonstrated effectiveness and good safety profile (high quality of evidence; strong recommendation).

a. Absolute contraindications: liver failure (high quality of evidence, strong recommendation).

b. Relative contraindications and cautions: hepatic insufficiency, chronic alcohol abuse or dependence (moderate quality of evidence, strong recommendation).

c. Maximum daily recommended dosages of $4 \mathrm{~g}$ per $24 \mathrm{~h}$ are not to be exceeded and must include "hidden sources" such as from combination pills (moderate quality of evidence, strong recommendation).

II. Nonselective NSAIDs and COX-2 selective inhibitors may be rarely considered and with extreme caution in highly selected individuals (high quality of evidence, strong recommendation).

a. Patient selection: other (safer) therapies have failed; evidence of continuing therapeutic goals not met; ongoing assessment of risks and complications outweighed by therapeutic benefits (low quality of evidence, strong recommendation).

b. Absolute contraindications: current active peptic ulcer disease (low quality of evidence, strong recommendation), chronic kidney disease (moderate level of evidence, strong recommendation), heart failure (moderate level of evidence, weak recommendation).

c. Relative contraindications and cautions: hypertension, Helicobacter pylori, history of peptic ulcer disease, concomitant use of corticosteroids or SSRIs (moderate quality of evidence, strong recommendation).

III. Older persons taking nonselective NSAIDs should use a proton pump inhibitor or misoprostol for gastrointestinal protection (high quality of evidence, strong recommendation).

IV. Patients taking $\mathrm{COX}-2$ selective inhibitors with aspirin should use a proton pump inhibitor or misoprostol for gastrointestinal protection (high quality of evidence, strong recommendation).

V. Patients should not take more than one nonselective NSAID or $\mathrm{COX}-2$ selective inhibitor for pain control (low quality of evidence, strong recommendation).

VI. Patients taking aspirin for cardioprophylaxis should not use ibuprofen (moderate quality of evidence, weak recommendation).

VII. Patients taking nonselective NSAIDs and COX-2 selective inhibitors should be assessed routinely for gastrointestinal and renal toxicity, hypertension, heart failure, and other drug-drug and drug-disease interactions (weak quality of evidence, strong recommendation).

\section{B. Opioids}

VIII. Patients with moderate to severe pain, painrelated functional impairment, or diminished quality of life because of pain may be considered for opioid therapy (low quality of evidence, strong recommendation).

IX. Patients with frequent or continuous pain on a daily basis may be treated with around-the-clock timecontingent dosing aimed at achieving steady-state opioid therapy (low quality of evidence, weak recommendation). X. Clinicians should anticipate, assess for, and identify potential opioid-associated adverse effects (moderate quality of evidence, strong recommendation).

XI. Maximal safe doses of acetaminophen or NSAIDs should not be exceeded when using fixeddose opioid combination agents as part of an analgesic 
regimen (moderate quality of evidence, strong recommendation).

XII. When long-acting opioid preparations are prescribed, breakthrough pain should be anticipated, assessed, and prevented or treated using short-acting immediate-release opioid medications (moderate quality of evidence, strong recommendation).

XIII. Clinicians well versed in the use and risks of methadone should initiate it and titrate it cautiously (moderate quality of evidence, strong recommendation). XIV. Patients taking opioid analgesics should be reassessed for ongoing attainment of therapeutic goals, adverse effects, and safe and responsible medication use (moderate quality of evidence, strong recommendation).

\section{Adjuvant analgesic drugs}

XV. All patients with neuropathic pain are candidates for adjuvant analgesics (strong quality of evidence, strong recommendation).

XVI. Patients with fibromyalgia are candidates for a trial of approved adjuvant analgesics (moderate quality of evidence, strong recommendation).

XVII. Patients with other types of refractory persistent pain may be candidates for certain adjuvant analgesics (i.e., back pain, headache, diffuse bone pain, temporomandibular disorder) (low quality of evidence, weak recommendation).

XVIII. Tertiarytricyclicantidepressants(amitriptyline, imipramine, doxepin) should be avoided because of higher risk for adverse effects (i.e., anticholinergic effects, cognitive impairment) (moderate quality of evidence, strong recommendation).

XIX. Adjuvant agents may be used alone, but often the effects are enhanced when used in combination with other pain analgesics and nondrug strategies (moderate quality of evidence, strong recommendation).

XX. Therapy should begin with the lowest possible dose and increase slowly based on response and side effects, with the caveat that some agents have a delayed onset of action and therapeutic benefits are slow to develop. For example, gabapentin may require 2 to 3 weeks for onset of efficacy (moderate quality of evidence, strong recommendation).

XXI. An adequate therapeutic trial should be conducted before discontinuation of a seemingly ineffective treatment (weak quality of evidence, strong recommendation).

\section{Other drugs}

XXII. Long-term systemic corticosteroids are reserved for patients with pain-associated inflammatory disorders or metastatic bone pain. Osteoarthritis should not be considered an inflammatory disorder (moderate quality of evidence, strong recommendation).

XXIII. Patients with localized neuropathic pain are candidates for topical lidocaine (moderate quality of evidence, strong recommendation).

XXIV. Patients with localized nonneuropathic pain may be candidates for topical lidocaine (low quality of evidence, weak recommendation).
XXV. Patients with other localized nonneuropathic persistent pain may be candidates for topical NSAIDs (moderate quality of evidence, weak recommendation).

XXVI. Other topical agents including capsaicin or menthol may be considered for regional pain syndromes (moderate quality of evidence, weak recommendation).

XXVII. Many other agents for specific pain syndromes may require caution in older persons and merit further research (i.e., glucosamine, chondroitin, cannabinoids, botulinum toxin, alpha- 2 adrenergic agonists, calcitonin, vitamin $\mathrm{D}$, bisphosphonates, ketamine) (low quality of evidence, weak recommendation).

The WHO developed an approach to cancer pain management that may be applied to management of geriatric pain because of their significant overlap. To manage pain effectively, the WHO recommends oral administration of analgesics "around the clock" (determined regular intervals), individualized for the patient, and following the "analgesic ladder." This treatment is inexpensive and estimated to be $80 \%$ to 90\% effective (Kaye et al., 2010; Tapsell et al., 2006). According to this approach:

1. For mild pain conditions, acetaminophen is the most appropriate first choice because it has the least known side effects.

2. For mild to moderate pain conditions or pain uncontrolled with acetaminophen, NSAIDs are the most appropriate.

3. For pain initially rated moderate or pain refractive to NSAID treatment, a weaker opioid (i.e., codeine) is the most appropriate first choice. Other weak opioids include hydrocodone, propoxyphene, and oxycodone in combination with acetaminophen.

4. For pain initially rated as severe or pain refractory to the previously mentioned weaker opioids, an opioid agonist (i.e. morphine) is chosen. Other opioid agonist drugs include hydromorphone, fentanyl, methadone, and levorphanol.

5. Adjuvant medication may be used for pain and anxiety relief and for synergism with medications mentioned above.

\section{Adujvants}

Adjuvant drugs are non-analgesics that may enhance the efficacy of other analgesics, independently provide analgesia for specific types of pain, or treat certain symptoms of pain. Adjuvant drug therapy is necessary to increase the analgesic effects of other medications. Regiments of multiple drugs may be implemented before the optimal treatment combination for each individual is obtained. Common adjuvant drugs used in pain treatments include but are not limited to the medications listed in Box 2. 


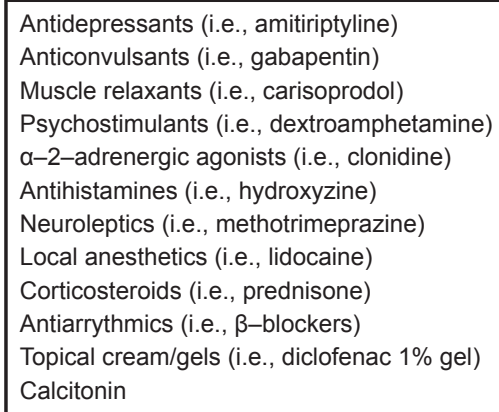

Box 2. Common drugs in the geriatric population

Modified from Kaye A. D., Baluch A., Scott J. T. (2010). Pain management in the elderly population: A Review. The Ochsner Journal, 10, 185.

Studies are currently being conducted to evaluate the efficacy of herbs and supplements in the treatment of pain. For example, Level II evidence supports that ginger in studied dose ranges from $510 \mathrm{mg}$ to $1 \mathrm{~g}$ per day reduces osteoarthritis and rheumatoid arthritis, including arthritic knee pain, ranking slightly below ibuprofen. Further, anti-inflammatory effects are known for omega fatty acids. To determine how these modalities compare with current drug therapies, more studies and comparison trials are required (Tapsell et al., 2006).

\section{Physical therapy and alternative modalities}

rehabilitation allows geriatric pain patients to live a more functional and independent life as a result of their adapting to the loss of psychological, physical, or social skills. Individual-specific treatment plans are created to minimize pain and enhance function. ADL assessments are used to evaluate the level of patient disability and guide treatment. The objectives of the rehabilitation process include stabilizing the primary order, preventing secondary disabilities, decreasing pain perception using a multidisciplinary approach, treating deficits in function, and promoting overall adaption to the disability and life demands (Brummel-Smith, 1997). The beneficial effects of spinal manipulative therapy and acupuncture on the attenuation of pain sensation have been established by recent studies; however, their effects in elderly populations remain to be elucidated (Bialosky, Bishop, Robinson, Zeppieri, \& George, 2009; Lang, Stoer, Schober, Audette, \& Irnich, 2010).

\section{Therapeutic Exercises}

Therapeutic exercises are used to improve or to maintain strength. They also help to maintain flexibility, prevent contractures, improve joint range of motion (ROM), and improve both proprioception and balance. Some classifications of these modalities include passive, active, active-assisted, resistive exercises, flexibility (stretching) exercises, and aerobic conditioning (Javier \& Montagnini, 2011). Devices used during therapy may include rowing machines, ergometers, and treadmills. An example of a therapeutic regimen may be any resistive exercise whereby the patient performs a set of $8-12$ repetitions at $60-70 \%$ of one maximal rep over a 12-week program in the affected portion of the body. Studies have shown that such a regimen may improve quality of life, decrease fatigue, and increase strength (Segal et al., 2003).

\section{Physical modalities}

Physical modalities for pain control may contribute to improved physical function and may include cold, heat, massage, ultrasound, and transcutaneous electrical nerve stimulation (TENS), among others. Cryotherapy may be applied to the affected areas to reduce blood flow, metabolism, swelling, and muscle spasms that consequentially decrease nerve activity and pain. Application of cryotherapy should be limited to less than $30 \mathrm{~min}$ at a temperature $\sim 59^{\circ} \mathrm{F}\left(15^{\circ} \mathrm{C}\right)$ and should be used with caution or not at all in patients with nerve damage or perfusion problems. Heat may also be employed via a hot pack, hot water bottle, or heating pad with temperatures ranging from $109-113^{\circ} \mathrm{F}(43-$ $45^{\circ} \mathrm{C}$ ) for $3-30 \mathrm{~min}$ at a time. The resultant vasodilation increases the supply of oxygen and nutrients to the site while simultaneously eliminating carbon dioxide and metabolic waste. It is especially useful for muscle spasms, myalgia, contractures, bursitis, and fibromyalgia.

Massage therapy, one of the most common forms of manual therapy, may be used to mobilize a joint, stretch adhesions, reduce swelling, and mobilize fluids by manipulating the surface of the body mechanically. Generally, a physical therapist or massage therapist will apply this technique by stroking, compressing, tapping, or using sustained pressure on the affected soft tissue. The most commonly used form of electrical delivery is transcutaneous electrical nerve stimulation (TENS). Small pads with conducting gel may be applied to the painful area, which eventually lead to changes in perfusion, improved healing, and restoration of proper nerve signaling.

\section{Acupuncture}

One of the oldest healing techniques, acupuncture, is part of traditional Chinese medicine and has become more prevalent in the Western hemisphere. Practitioners will stimulate specific points on the body that often have no anatomic correlation or relationship to the nervous system. Stimulation is via insertion of thin needles through the skin in an attempt to regulate the flow of "qi," vital energy, along pathways referred to as meridians. Acupuncture has been used to help treat common ailments in the geriatric population including back pain, joint pain, neck pain, severe headaches, and recurring pain. Accupuncture stimulation has a resultant increase in tissue vasodilation for several hours, mediating or modulated by a number of chemicals being released or activated. These include, among others, acetylcholine, endorphins, norepinephrine, dopamine and 5-hydroxytryptamine.

Currently, results for acupuncture versus other treatments (NSAIDS, lidocaine injections, manual 
manipulation) have been mixed (Furlan et al., 2008). Further randomized clinical studies with larger study populations must be undertaken to determine effectiveness of this modality compared to others currently available.

\section{Psychological modalities}

Current psychological modalities utilized to manage chronic pain attempt to achieve increased self-management, behavioral change, and cognitive change instead of focusing directly on the actual locus of pain. Psychological interventions target emotional, behavioral, and cognitive aspects of chronic pain with some of the more frequently employed modalities including psychophysiological techniques, behavioral modification, cognitive behavioral therapy, and acceptance-based interventions.

Psychophysiological techniques such as biofeedback allow for patients to learn to interpret feedback in the form of physiological data based on certain physiological functions. For example, equipment may be used to recognize areas of body tension so the patient can subsequently learn to relax those areas. Feedback may come in the form of blood pressure, muscle tone, heart rate, skin temperature, or brain electrical activity. These modalities have been shown to be efficacious and have shown significant improvements in certain conditions (Nestoriuc \& Martin, 2007).

Behavioral approaches may include relaxation techniques such as breathing exercises, visualization/ guided imagery, and graded activation. Collectively these techniques have shown to be efficacious for acute and chronic pain quality of life (Baird \& Sands, 2006; Carroll \& Seers, 1998; Morone \& Greco, 2007). However, these techniques are not necessarily applicable to individuals with significant cognitive impairment or severe depression (Kaye et al., 2010; Norelli \& Harju, 2008).

The presence of depression and/or anxiety in patients can affect abilities to improve the quality of life and manage pain; therefore, co-morbid mental disorders can be addressed using psychotherapy, medication, or meditation. Socio-environmental factors may be adjusted to help patients cope with pain. A solid support system consisting of involved relatives and caregivers should be established as results in pain reduction.

\section{Interventional modalities}

Interventional modalities are invasive treatments used for a number of reasons and are important in pain management. First, these interventions assist to determine the precise cause of pain, which consequently helps physicians to make a more accurate diagnosis. Second, they remain a mainstay of chronic pain treatment, even though many of the procedures suffer from lack of research-based validity related to the difficulty of obtaining prospective randomized controlled studies. In this regard, placebo-controlled trials are difficult to obtain secondary to ethical considerations and patient enrollment challenges. Interventions generally target the actual neural structure involved in pain generation. Mechanisms of action vary but include infiltration with local anesthetics, steroids, opioid agents, and/or adjuvant agents, spinal cord stimulation, or ablation with either radiofrequency or neurolytic agents.

Intraspinal opioid therapy may be used to enhance efficacy and to minimize systemic side effects when compared to oral medication. Delivery may be via programmable, implanted pumps, accessible reservoir systems, or tunneled, exteriorized catheters. Studies performed have demonstrated improved analgesic efficacy and reduced toxic side effects when compared to patients who are on high dose oral opioid treatment (Smith et al., 2003; Rauck et al., 2003).

Neurolytic blockade is commonly used for endstage cancer pain related to potential neurodestruction from either phenol or alcohol. An example would be a celiac plexus neurolytic block for terminal intraabdominal cancer. The ganglia are situated in the retroperitoneal space and the approach, in general, has been reserved for severe pain in terminal end of life patients. This neurolytic block has been shown to significantly reduce pain, reduce opioid-related side effects, and increase quality of life (de Oliveira, dos Reis, \& Prado, 2004).

"Gate theory" proposed that the balance of small and large neural fiber firing influences the sensation of pain. Retrograde stimulation of large fibers would cause "closing" of the gate and thereby provoke nonpainful stimulation. Currently, pain stemming from the periphery and ischemic pain sites are the most common reasons for use of nerve stimulation for pain management. Complex regional pain syndrome and chronic radicular pain are examples of indications for nerve stimulation techniques. Statistically significant reductions in pain severity have been found with this treatment as well as marked reduction in analgesic requirements (Harke, Gretenkort, Ladleif, \& Rahman, 2005; Kemler, de Vet, Barendse, van den Wildenberg, \& van Kleef, 2006; Calvillo, Racz, Didie, \& Smith, 1998; Ebel, Balogh, \& Klug, 2004; Oakley \& Weiner, 1999).

Spinal pain is the most rampant noncancer pain syndrome and physicians have used radiofrequency to mitigate symptoms. Radiofrequency ablation has been used to attack pain stemming from the facet joint, which are paired synovial joints formed by the inferior articular process of one vertebra and the superior articular process of the vertebrae below. Two studies have demonstrated long-lasting efficacy with this procedure where percutaneous radiofrequency neurotomy was performed (Lord, Barnsley, Wallis, McDonald, \& Bogduk, 1996; Van Kleef et al., 1999).

Other studies dealing with facet-type pain have shown little benefit diagnostically or therapeutically with intra-articular facet joint injection with local anesthetic and steroid (Carette et al., 1991; Jackson, Jacobs, \& Montesano, 1988).

It should be noted that literature support for transforaminal nerve root injection, epidural steroid 
Table 1. Numerous liver changes observed in the elderly population

\begin{tabular}{ll}
\hline Size & Liver mass decreases by $1 \%$ per year after age 50 \\
Blood flow and velocity & $33 \%$ decrease in flow and $21 \%$ decrease in portal blood velocity over age 65 \\
Liver function & No significant changes in liver function tests \\
Conjugation & Not significantly changed \\
Microsomal hydroxylation/oxidation & Not significantly changed \\
Nonmicrosomal oxidation & Not significantly changed \\
Demethylation & Decreased with aging, caution with benzodiazepine use \\
Protein synthesis & Slightly decreased serum albumin levels, reduced quality of albumin, increased free fraction \\
Serum and biliary cholesterol & of protein bound drugs \\
Liver regeneration and capacity & Increased change of cholelithiasis and atherosclerosis \\
\hline
\end{tabular}

Modified from Lynch, D. (2000). Geriatric pain. In: P. Raj (Ed.), Practical Management of Pain. [3 ${ }^{\text {rd }}$ ed.] St. Louis, MO: Mosby, 278.

Table 2. Numerous kidney changes observed in the elderly population

\begin{tabular}{ll}
\hline Size & Kidney decreased in size by $20 \%-30 \%$ by age 70 \\
Renal tubules & Decreased length, number, and thickness \\
Interstitial tissue, tubular diverticula of renal tubules & Increased \\
Renal blood flow & Decreased by $10 \%$ per decade after age 20 \\
Glomerular filtration rate & Decreased $10 \mathrm{cc} / \mathrm{min} / \mathrm{decade}$ \\
Free water absorption & Decreased by $5 \%$ per decade after age 50 \\
\hline $\begin{array}{l}\text { Modified from Kielstein, J. T., Bode-Boger, S. M., Haller, H., \& Lynch, D. F. (2003). Functional changes in the ageing kidney: is there a role for asymmetric } \\
\text { dimethylarginine? Nephrology Dialysis Transplant, 18, 1245-1248, and Lynch, D. (2000). Geriatric pain. In: P. Raj (Ed.), Practical Management of Pain. [3rd ed.] }\end{array}$
\end{tabular}
St. Louis, MO: Mosby, 278.

injection, and sacroiliac joint injection has been established and is generally accepted (Manchikanti, Cash, McManus, Pampati, \& Benyamin, 2010a; Manchikanti et al., 2010b, Buenaventura, Datta, Abdi, \& Smith, 2009; Manchikanti, Singh, Falco, Cash, \& Pampati, 2010c, Rubinstein \& van Tulder, 2008). Radiofrequency of the sacroiliac joint has not been firmly established. It is clear, however, that information gained from nerve blocks may help with several aspects of pain control including the diagnosis of the precise pain generator and the overall prognosis of the pain state.

Pain injections play a role in the elderly with regard to preemptive analgesia, which involves blocking pain pathways before surgical incision or damage to tissue. Finally, interventional pain interventions can play a definitive therapeutic role in numerous common and atypical pain states. Further studies must be conducted in order to determine which patients may benefit from each type of interventional technique. However, the preponderance of evidence suggests a critical and important role for interventional pain procedures in all populations for many pain states, in particular in the geriatric population.

\section{Summary}

Persistent pain is prevalent in the elderly population, although it is not an inevitable part of aging (Kaye et al., 2010). It is important to understand how to manage pain effectively in old age, particularly because an increasing number of individuals are becoming older or living longer. Several problems, less common in younger adults, may complicate the treatment of pain. An accurate pain assessment is required for the most efficient strategy of pain treatment. Challenges to an effective pain assessment include pain underreporting by patients, atypical manifestations of pain in elderly, age-associated pharmacodynamic and pharmacokinetic changes to specific drugs, other general age-related changes, and misconceptions about tolerance or addiction to opioids. Pain specialists can be invaluable to the diagnosis and treatment of many pain states common in the elderly population (Kaye et al., 2010). In summary, physicians are able to provide geriatric patients with appropriate analgesia by using comprehensive assessment involving a multidisciplinary approach and the appropriate use of various treatment modalities.

\section{References}

American Geriatrics Panel on chronic pain in older persons (1998). The management of chronic pain in older persons. Journal of the American Geriatrics Society, 46, 635-651.

American Geriatrics Society Panel on Pharmacological Management of Persistent Pain in Older Persons (2009). Pharmacological management of persistent pain in older persons. Journal of the American Geriatrics Society, 57(8), 1331-1346.

Badley, E. M., \& Tennant, A. (1992). Changing profile of joint disorders with age: findings from a postal survey of the population of Calderdale, West Yorkshire, United Kingdom. Annals of the Rheumatic Diseases, 51, 366-371.

Baird, C. L., \& Sands, L. (2006). Effect of guided imagery with relaxation on health-related quality of life in older women with osteoarthritis. Research in Nursing Health, 29(5), 442-451.

Benesh, L. R., Szigeti, E., Ferraro, F. R., \& Gullicks J. N. (1997). Tools for assessing chronic pain in rural elderly women. Home Healthcare Nurse, 15, 207-211.

Berdine, H., \& Mihalyo, M. G. (2004). Demographics, pharmacoeconomics, assessment, and treatment of pain in the elderly. Journal of Pharmacy Practice, 17(2), 115-128.

Bialosky, J. E., Bishop, M. D., Robinson, M. E., Zeppieri, G. Jr., \& George, S. Z. (2009). Spinal manipulative therapy has an immediate effect on thermal pain sensitivity in people with low back pain: a randomized controlled trial. Physical Therapy, 89(12), 1292-1303.

Bresler, R., \& Bahl, J. J. (2003). Principles of drug therapy for the elderly patient. Mayo Clinical Proceedings, 78, 1564-1577. 
Broe, G. A. (1989). The neuroepidemiology of old age. In R. Tallis (Ed.), The Clinical Neurology of Old Age (pp. 50-80). Chichester, UK: Wiley.

Bruckenthal, P. (2008). Assessment of pain in the elderly adult. Clinics in Geriatric Medicine, 24(2), 213-236.

Brummel-Smith, K. (1997). Rehabilitation. In C. K. Cassel, H. J. Cohen, \& E. B. Larson (Eds.) Geriatric Medicine (pp. 211-226), [ed. 3]. New York: Springer.

Buenaventura, R. M., Datta, S., Abdi, S., \& Smith, H. S. (2009). Systematic Review of therapeutic transforaminal epidural steroid injections. Pain Physician, 12, 233-251.

Calvillo, O., Racz, G., Didie, J., \& Smith, K.(1998). Neuroaugmentation in the treatment of complex regional pain syndrome of the upper extremity. Acta Orthopedics Belgium, 64, 57-62.

Carette, S., Marcoux, S., Truchon, R., Grondin, C., Gagnon, J., Allard, Y., \& Latulippe, M. (1991). A controlled trial of corticosteroid injections into facet joints for chronic low back pain. New England Journal of Medicine, 325, 1002-1007.

Carroll, D., \& Seers, K. (1998). Relaxation for the relief of chronic pain: a systematic review. Journal of Advances in Nursing, 27(3), 476-487.

Chakour, M. C., Gibson, S. J., Bradbeer, M., \& Helme, R. D. (1996). The effect of age on $\mathrm{A} \delta$ - and $\mathrm{C}$-fibre thermal pain perception. Pain, 64(1), 143-152.

Charette, S. \& Ferrell, B. (2007). Rheumatic diseases in the elderly: Assessing chronic pain. Rheum Dis Clin North Am, 33(1), 109-122.

Cleeland, C. S. (1998). Undertreatment of cancer pain in elderly patients. Journal of the American Medical Association, 279, 1914 1915.

Coleman, P. D., \& Flood, D. G. (1987). Neuroa numbers and dendritic extent in normal aging and Alzheimer's disease. Neurobiology of Aging, 8, 521.

Crook, J., Rideout, E., \& Browne, G. (1984). The prevalence of pain complaints in a general population. Pain, 18, 299-314.

Crooks, J., \& Stevenson, I. H. (1981). Drug response in the elderlysensitivity and pharmacokinetic considerations. Age Ageing, 10(2), 73.

de Oliveira, R., dos Reis, M. P., \& Prado, W.,A. (2004). The effects of early or late neurolytic sympathetic plexus block on the management of abdominal or pelvic cancer pain. Pain, 110, 400-408.

Ebel, H., Balogh, A., \& Klug, N. (2004). Augmentative treatment of chronic deafferent pain syndromes after peripheral nerve lesions. Minim Invas Neurosurg, 43, 44-50.

ElDesoky, E. (2007). Pharmacokinetic-pharmacodynamic crisis in the elderly. American Journal of Therapeutics, 14(5), 488-498.

Ferrell, B. A. (1991). Pain management in elderly people. Journal of the American Geriatrics Society, 39, 64-73.

Ferrell, B. A., Ferrell, B. R., \& Rivera, L. M. (1995). Pain in cognitively impaired nursing home patients. Journal of Pain and Symptom Management, 10(8), 591-598.

Ferrell, B. (1996) Overview of aging and pain. In B. Ferrell \& B. Ferrell (Eds.), Pain in the Elderly (pp. 1-10). Seattle: IASP Press.

Ferrell, B. (2000). Pain management. Clinics in Geriatric Medicine, 16(4), 853-873.

Furlan, A. D., Yazdi, F., Tsertsvadze, A., Gross, A., Van Tulder, M., Santaguida, L., Gagnier, J., Ammendolia, C., Dryden, T., Doucette, S., Skidmore, B., Danie, R., Yucha, C., \& Montgomery, D. (2008). Evidence-based practice in biofeedback and neurofeedback. Wheat Ridge, CO: AAPB and Nestoriuc, Y., Martin, A. Efficacy of biofeedback for migraine: a meta-analysis. Pain. 2007, 128 (1-2), $111-127$.

Gagliese, L., \& Melzack, R. (1997). Age differences in the quality of chronic pain: A preliminary study. Pain Research and Management, $2,157-162$.

Gagliese, L. (2002). Assessment of pain in the elderly. In D. C. Turk \& R. Melzack (Eds.), Handbook of pain assessment (pp. 119-133). New York, NY: Guilford Press.

George, J., Elliott, R. A., \& Stewart, D. C. (2008). A systematic review of interventions to improve medication taking in elderly patients prescribed multiple medications. Drugs Aging, 25(4), 307-324.

Ghafoor, V. (2003). Management of painful conditions in the elderly. Journal of Pharmacy Practice, 16(4), 276-283.

Gibson, S. J. (2003). Pain and aging: the pain experience over the adult lifespan. In J. O. Dostrovsky, D. B. Carr, \& M. Koltzenburg
(Eds.), Proceedings of the 10th World Congress on Pain (pp. 767790). Seattle: IASP.

Harke, H., Gretenkort, P., Ladleif, H., \& Rahman, S. (2005). Spinal cord stimulation in sympathetically maintainted complex pain syndrome type I with severe disability. A prospective study. European Journal of Pain, 9, 363-373.

Herr, K. A., \& Mobily, P. R. (1993). Comparison of selected pain assessment tools for use with the elderly. Applied Nursing Research, 6, 39-46.

Herr, K. A., \& Garand, L. (2001). Assessment and measurement of pain in older adults. Clinics Geriatric Medicine, 17(3), 457-478.

Herr, K. A., Spratt, K., Mobily, P. R., \& Richardson, G. (2004). Pain intensity assessment in older adults: use of experimental pain to compare psychometric properties and usability of selected pain scales with younger adults. Clin J Pain, 20, 207-19.

Jackson, R. P., Jacobs, R. R., \& Montesano, P. X. (1988). Facet joint injection in low back pain. Spine, 13, 966-971.

Javier, N. S., \& Montagnini, M. L. (2011). Rehabilitation of the Hospice and Palliative Care Patient. Journal of Palliative Medicine, 14(5), 638-648.

Kaye, A. D., Baluch, A., Scott, J. T. (2010). Pain management in the elderly population: A review. The Ochsner Journal, 10, 179-187.

Kemler, M. A., de Vet, H. C., Barendse, G. A., van den Wildenberg, F. A., \& van Kleef, M. (2006). Spinal cord stimulation for chronic reflex sympathetic dystrophy-five year follow-up. New England Journal of Medicine, 354, 2394-2396.

Kinirons, M. T., \& Crome, P. (1997). Clinical pharmacokinetic considerations in the elderly: an update. Clinical Pharmacokinetics, $33,302-312$.

Kitani, K. (1986). Hepatic drug metabolism in the elderly. Hepatology, 6, 316-319.

Kohli, H. S., Bhat, A., Aravindan, S. K., Jha, V., Gupta, K. L., \& Sakhuja, V. (2006). Spectrum of renal failure in elderly patients. International Urology and Nephrology, 38(3-4), 759-765.

Kovach, C., Weissman, D., Griffie, J., Matson, S., \& Muchka, S. (1999). Assessment and treatment of discomfort for people with late-stage dementia. Journal of Pain and Symptom Management, 18(6), 412-419.

Lautenbacher, S., Kunz, M., Strate, P., Nielsen, J., \& ArendtNielsen, L. (2005). Age effects on pain thresholds, temporal summation and spatial summation of heat and pressure pain. Pain, $115,410-418$.

Lin, Y. H., Hsieh, S. C., Chao, C. C., Chang, Y. C., \& Hsieh, S. T. (2005). Influence of aging on thermal and vibratory thresholds of quantitative sensory testing. Journal of the Peripheral Nervous System, 10, 269-281.

Lin, W. C., Lum, T. Y., Mehr, D. R., \& Kane, R. L. (2006) Measuring pain presence and intensity in nursing home residents. Journal of the American Medical Directors Association, 7, 147-153.

Lang, P. M., Stoer, J., Schober, G. M., Audette, J. F., \& Irnich, D. (2010). Bilateral acupuncture analgesia observed by quantitative sensory testing in healthy volunteers. Anesthesia and Analgesia, 110(5), 1448-1456.

Long, D. M. (1985). Aging in the nervous system. Neurosurgery, 17, 348-354.

Lord, S. M., Barnsley, L., Wallis, B. J., McDonald, G. J., \& Bogduk, N. (1996). Percutaneous radio-frequency for chronic cervical zygapophyseal-joint pain. New England Journal of Medicine, 335, 1721-1726.

Manchikanti, L., Singh, V., Datta, S., Cohen, S. P., \& Hirsch, J. A. (2009). Comprehensive review of epidemiology, scope, and impact of spinal pain. Pain Physician, 12(4), 35-70.

Manchikanti, L., Cash, K. A., McManus, C. D., Pampati, V., \& Benyamin, R. M. (2010a). Preliminary results of a randomized, double-blind, controlled trial of fluoroscopic lumbar interlaminar epidural injections in managing chronic lumbar discogenic pain without disc herniation or radiculitis. Pain Physician, 13, 279292.

Manchikanti, L., Datta, S., Gupta, S., Munglani, R., Bryce, D. A., Ward, S. P., Benyamin, R. M., Sharma, M. L., Helm, S. 2nd, Fellows, B., \& Hirsch, J. A. (2010b). A critical review of the American Pain Society clinical practice guidelines for interventional techniques: part 2. Therapeutic interventions. Pain Physician, 13(4), 215-264. 
Manchikanti, L., Singh, V., Falco, F. J., Cash, K. A., \& Pampati, V. (2010c). Evaluation of the effectiveness of lumbar interlaminar epidural injections in managing chronic pain of lumbar disc herniation or radiculitis: a randomized, double-blind, controlled trial. Pain Physician, 13, 343-355.

Melzack, R. (1975). The McGill Pain Questionnaire: appraisal and current status. In N. E. Morone, \& C. M. Greco (2007), Mind-body interventions for chronic pain in older adults: a structured review. Pain Medicine, 8(4), 359-375.

Morone, N. E., \& Greco, C. M. (2007). Mind-body interventions for chronic pain in older adults: a structured review. Pain Medicine, 8(4), 359-375.

Muravchick, S. (1998). The effects of aging on anesthetic pharmacology. Acta Anaesthesiology Belgium, 49(2), 79-84.

Nestoriuc, Y., \& Martin, A. (2007). Efficacy of biofeedback for migraine: a metal-analysis. Pain, 128(1-2), 111-127.

Norelli, L., \& Harju, S. K. (2008). Behavioral approaches to pain management in the elderly. Clinics in Geriatric Medicine, 24(2), 335-342.

Oakley, J., \& Weiner, R. L. (1999). Spinal cord stimulation for complex regional pain syndrome: a pro-spective study of 19 patients at 2 centers. Neuromodulation, 2, 47-50.

Parmelee, P. A., Smith, B., \& Katz, I. R., (1993). Pain complaints and cognitive status among elderly institution residents. Journal of the American Geriatrics Society, 41(5), 517-522.

Pautex, S., Herrmann, F., Le Lous, P., Fabjan, M., Michael, J. P., \& Gold, G. (2005). Feasibility and reliability of four pain selfassessment scales and correlation with an observational rating scale in hospitalized elderly demented patients. Journal of Gerontology Series A: Biological Sciences and Medical Sciences, 60, 524-529.

Pautex, S., Michon, A., Guedira, M., Emond, H., Le Lous, P., Samaras, D., Michel, J. P., Herrmann, F., Giannakopoulos, P., \& Gold, G. (2006). Pain in severe dementia: self-assessment or observational scales? Journal of the American Geriatrics Society, 54, 1040-1045.

Pickering, G., Jourdan, D., Eschalier, A., \& Dubray, C. (2002). Impact of age, gender and cognitive functioning on pain perception. Gerontology, 48(2), 112-118.

Rauck, R. L., Cherry, D., Boyer, M. F., Kosek, P., Dunn, J., \& Alo, K. (2003). Long-term intrathecal opioid therapy with a patientactivated, implanted delivery system for the treatment of refractory cancer pain. Journal of Pain, 4, 441-447.

Rehman, H. U., \& Masson, E. A. (2001). Neuroendocrinology of ageing. Age Ageing, 30, 279-287.

Rubinstein, S. M., \& van Tulder, M. (2008). A best-evidence review of diagnostic procedures for neck and low-back pain. Best Practices in Research in Clinical Rheumatology, 22(3), 471-482.

Samorajski, T. (1976). How the human brain responds to aging. Journal of the American Geriatrics Society, 24, 4-11.

Segal, R. J., Reid, R. D., Courneya, K. S., Malone, S. C., Parliament, M. B., Scott , C. G., Venner, P. M., Quinney, H. A., Jones, L. W., D'Angelo, M. E., \& Wells, G. A. (2003). Resistance exercise in med receiving androgen deprivation therapy for prostate cancer. Journal of Clinical Oncology, 21, 1653-1659.
Sengstaken, E. A., \& King, S. A. (1993). The problems of pain and its detection among geriatric nursing home residents. Journal of the American Geriatrics Society, 41, 541-544.

Shi, S., Mörike, K., \& Klotz, U. (2008). The clinical implications of ageing for rational drug therapy. European Journal of Clinical Pharmacology, 64(2), 183-199.

Simon, R. P., Aminoff, J. J., \& Greenberg, D. A. (1989). Clinical Neurology. Norwalk, Conn, Appleton and Lange. In D. C. Turk \& R. Melzack (Eds.), Handbook of pain assessment (pp. 35-52). New York, NY: Guilford Press.

Smith, T. J., Staats, P. S., Deer, T., Stearns, L. J., Rauck, R. L., BoortzMarx, R. L., Buchser, E., Català, E., Bryce, D. A., Coyne, P. J., \& Pool, G. E.; Implantable Drug Delivery Systems Study Group (2003). Randomized clinical trial of an implantable drug delivery system compared with comprehensive medical management for refractory cancer pain: impact on pain, drug related toxicity, and survival. Journal of Clinical Oncology, 20(19), 4040-4049.

Steinman, M. A., \& Hanlon, J. T. (2010). Managing mediations in clinically complex elders: "There's got to be a happy medium." Journal of the American Medical Association, 304(14), 1592-1601.

Stiff, J. L. (1993). Evaluations of the geriatric patient. In M. C. Rogers, B. G. Covino, J. H. Tinker (Eds.), Principles and Practice of Anesthesiology (pp. 480-492.). St. Louis:Year Book.

Tapsell, L. C., Hemphill, I., Cobiac, L., Patch, C. S., Sullivan, D. R., Fenech, M., Roodenrys, S., Keogh, J. B., Clifton, P. M., Williams, P. G., Fazio, V. A., \& Inge, K. E. (2006). Health benefits of herbs and spices: the past, the present, the future. Medical Journal of Austria, 185(4), 4-24.

Turnheim, K. (2003). When drug therapy gets old: pharmacokinetics and pharmacodynamics in the elderly. Experimental Gerontology, $38,843-853$.

Van Kleef, M. V., Barendse, G. A., Kessels, A., Voets, H. M, Weber, W. E., \& de Lange, S. (1999). Randomized trial of radiofrequency lumbar facet denervation for chronic low back pain. Spine, 24, 1937-1942.

Verrillo, R. T., Bolanowski, S. J., Gescheider, G. A. (2002). Effect of aging on the subjective magnitude of vibration. Somatosensensory Motor Research, 19(3), 238-244.

Vuyk, J. (2003). Pharmacodynamics in the elderly. Best Practices in Research Clinical Anaesthesiology, 17, 207-218.

Warden, V., Hurley, A. C., \& Volicer, L. (2003). Development and psychometric evaluation of the Pain Assessment in Advanced Dementia (PAINAD) scale. Journal of the American Medical Directors Association, 4, 9-15.

Weiner, D. K., Peterson, B. L., Logue, P., \& Keefe, F. J. (1998). Predictors of self-report in nursing home residents. Aging Clinical and Experimental Research, 10, 411-420.

Woo, J., Ho, S. C., Lau, J., \& Leung, P. C. (1994). Musculoskeletal complaints and associated consequences in elderly Chinese aged 70 years and over. Journal of Rheumatology, 21, 1927-1931.

G. D., Siegelaub, A. B., \& Collen, M. F. (1972). Pain tolerance: Differences according to age, sex and race. Psychosomatic Medicine, 34(6), 548-556.

Wright, R. M., \& Warpula, R. W. (2004). Geriatric pharmacology: Safer prescribing for the elderly patient. Journal of the American Podiatric Medical Associaton, 94(2), 90-97. 